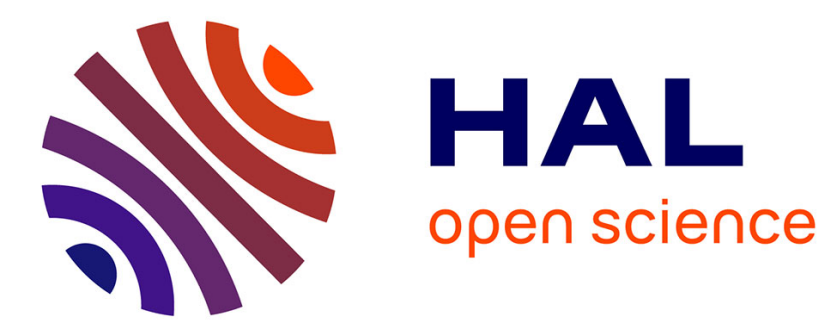

\title{
Regard anthropologique et historique sur l'adoption.
} Agnès Fine

\section{To cite this version:}

Agnès Fine. Regard anthropologique et historique sur l'adoption.. Informations sociales, 2008, Heurts et bonheur de l'adoption, 146, pp.8-19. hal-01278600

\section{HAL Id: hal-01278600 \\ https://hal.science/hal-01278600}

Submitted on 24 Feb 2016

HAL is a multi-disciplinary open access archive for the deposit and dissemination of scientific research documents, whether they are published or not. The documents may come from teaching and research institutions in France or abroad, or from public or private research centers.
L'archive ouverte pluridisciplinaire HAL, est destinée au dépôt et à la diffusion de documents scientifiques de niveau recherche, publiés ou non, émanant des établissements d'enseignement et de recherche français ou étrangers, des laboratoires publics ou privés.

\section{(1) $(1) \Theta$}

Distributed under a Creative Commons Attribution - NonCommercial - NoDerivatives| 4.0 
Agnès Fine

Directrice d'Etudes à l'EHESS

LISST-Centre d'anthropologie sociale

\section{Regard anthropologique et historique sur l'adoption}

\section{De quelques formes d'adoption dans des sociétés lointaines}

Les anthropologues qui ont travaillé sur les sociétés océaniennes ou africaines ont été frappés par le contraste entre l'adoption qui y est pratiquée et celle que nous connaissons dans nos sociétés ${ }^{1}$. Loin d'être une réponse ponctuelle aux défaillances de la parenté, enfants orphelins ou abandonnés d'un côté, couples stériles de l'autre, comme c'est le cas dans les sociétés occidentales, les adoptions s'y pratiquent avec une grande ampleur entre parents de naissance vivants et parents adoptifs féconds. Les anthropologues ont parfois eu des difficultés à différencier les adoptions (au caractère définitif) des placements provisoires (système de fosterage). Qu'elle soit limitée dans le temps ou définitive, la circulation des enfants apparait très fréquente dans un grand nombre de sociétés, et particulièrement en Afrique et en Océanie où on rencontre des parents qui élèvent avec leurs propres enfants un enfant qui leur a été donné tandis qu'un des leurs vit dans une autre famille. Les enfants, un bien précieux s'il en est, font ainsi l'objet de dons qui créent ou renforcent les liens entre donneurs et destinataires, les deux étant généralement de même niveau social.

Nombreuses sont les recherches anthropologiques sur les caractéristiques des adoptants (âge, place dans la parenté, lignage d'appartenance), celles des parents de naissance, celles des enfants adoptés, enfin les circonstances de l'adoption et ses conséquences juridiques et concrètes. Comme les pratiques varient beaucoup selon les diverses sociétés étudiées, il est impossible d'en présenter un tableau synthétique. Signalons cependant quelques traits assez répandus. L'enfant est le plus souvent adopté dans sa parenté : ses grands-parents tout d'abord qui semblent avoir souvent une sorte de «droit de préemption $»^{2}$ sur le premier enfant de leur fille, par un oncle, une tante, seul ou en couple, un frère ou une sœur réelle ou classificatoire, des cousins, parfois des voisins ou des proches. De sorte qu'il n'y a pas de véritable coupure de l'enfant adopté avec sa famille d'origine. Le plus souvent, l'enfant connaît ses parents de naissance et ses frères et sœurs avec lesquels il entretient des relations régulières et il use à leur égard des termes de parenté habituels.

Prenons l'exemple des Sulka de Nouvelle-Bretagne ${ }^{3}$. Le sexe n'intervient guère dans la décision d'adopter, pas plus que l'âge. Un enfant peut être adopté avant sa naissance comme au moment de sa puberté et tout le monde peut adopter tout le monde. «Un veuf âgé peut adopter un enfant qui lui portera assistance dans les tâches quotidiennes ; un célibataire peut adopter l'enfant d'un parent qui prévoit de s'absenter longtemps du village ; une grand-mère peut adopter le petitenfant dont elle a assuré le sevrage ; un couple peut adopter un enfant auquel il s'est attaché après en avoir eu la garde ; un adolescent peut adopter un enfant plus jeune ; un enfant, enfin peut être adopté à sa propre demande par les personnes chez qui il se trouvait en visite ${ }^{4}$. L'adoption se fait

\footnotetext{
${ }^{1}$ La bibliographie sur l'adoption en anthropologie comme en histoire est immense et ne peut apparaître dans ce texte. Je n'indiquerai donc que les références des études citées.

${ }^{2}$ Selon l'expression employée par J.Massard à propos de la Malaisie. Voir Massard , 1988, « Engendrer ou adopter : deux visions concurrentes de la parenté chez les Malais péninsulaires », Anthropologie et sociétés, 12,2, p 41-62 ; même constatation chez les Inuit, voir B.Saladin d'Anglure, « L'élection parentale chez les Inuit » in A.Fine (ed), 1998, Adoptions, Ethnologie des parentés choisies, Paris, Editions de la Maison des sciences de l'Homme, p 121-149

${ }^{3}$ Monique Jeudy-Ballini, « Naître par le sang, renaître par la nourriture : un aspect de l'adoption en Océanie », in A.Fine (éd), 1998, Adoptions. op.cit, p 19-44

${ }^{4}$ Monique Jeudy-Ballini, op.cit, p 23
} 
généralement à la demande de l'adoptant, demande que les parents peuvent difficilement refuser, quoiqu'il leur en coûte. Suzanne Lallemand qui a étudié l'adoption traditionnelle au Togo, a mis en évidence les rapports entre circulation des enfants et échange des femmes, les deux concourant à développer les alliances et donc les échanges de toutes sortes entre groupes ${ }^{5}$. Elle s'est interrogée également sur le fait de savoir si les dons d'enfants étaient plus fréquents dans les sociétés dont la terminologie de la parenté est la même pour des ascendants de même sexe, par exemple lorsqu'un enfant désigne comme père, non seulement son géniteur, le conjoint de sa mère, mais aussi les frères de son père ${ }^{6}$, ou bien lorsque les sœurs de sa mère sont désignées comme elle.

L'absence de secret et la poursuite des relations de l'enfant adopté avec sa famille de naissance contrastent avec les pratiques des sociétés occidentales dans lesquelles il n'existe généralement aucune relation entre parents de naissance et parents adoptifs, même si cela n'a pas toujours été le cas et même si les choses changent actuellement.

Pour mieux comprendre les logiques institutionnelles occidentales, on peut prendre l'exemple de la France et brosser à grands traits son histoire.

\section{Evolution historique de l'institution en France}

- de la recherche d'un descendant héritier...

Il faut tout d'abord insister sur l'absence remarquable de l'adoption comme institution juridique de filiation en Europe de l'Ouest depuis le haut Moyen Âge. Elle témoigne de l'hostilité de cette société à l'introduction d'un étranger au sang au sein d'une lignée. L'adoption n'apparaît dans le droit français qu'en 1804 avec le Code Civil. La référence explicite des juristes de l'époque est l'adoption antique, grecque et romaine, dont ils partagent l'esprit : l'institution ne devant pas concurrencer le mariage légitime, seules peuvent adopter les personnes sans enfant légitime, âgées de plus de 50 ans (âge auquel elles n'espèrent plus avoir de descendants naturels). Elles adoptent des enfants majeurs (25 ans) auxquels elles doivent avoir " dans leur minorité et pendant six ans au moins fourni des secours et donné des soins ininterrompus ». Elles leur transmettent leurs biens et leur nom, ce dernier devant figurer après celui du père naturel de l'adopté. En effet, les adoptés restent dans leur famille naturelle, ce qui signifie dans les faits qu'ils gardent leur droit à la succession de leurs parents et conservent leur nom d'origine. Tout au long du XIX ${ }^{\mathrm{e}}$ siècle, les adoptants (à peine une centaine de cas par an pour toute la France pendant presque tout le siècle) - sont dans leur majorité des possédants, propriétaires rentiers, commerçants, ou membres de professions libérales qui veulent transmettre leurs biens, dans la moitié des cas à leurs enfants naturels (reconnus ou non reconnus) qu'ils légitiment ainsi, et secondairement aux enfants de leur conjoint $^{7}$. Notons un fait important : les adoptés ne sont pas des enfants abandonnés. Ils ont généralement vécu avec un parent de naissance ou avec les deux qui eux-mêmes connaissent et estiment leurs parents adoptifs, l'adoption se faisant souvent dans la parenté ou parmi ses proches. Un couple sans enfant peut demander à un frère ou une sœur de l'un ou l'autre conjoint, ou à un ami de lui « donner » un de ses enfants ${ }^{8}$ en adoption.

\footnotetext{
${ }^{5}$ Suzanne Lallemand, 1993, La circulation des enfants en société traditionnelle. Prêt, don, échange, Paris, L'harmattan.

${ }^{6}$ Suzanne Lallemand, « Mes mères et mes pères. Rapports entre filiation, terme de parenté et adoptionfosterage », in I.Leblic(éd),2004, De l'adoption. Des pratiques de filiation différentes, Presses universitaires Blaise Pascal, p 299-321

${ }^{7}$ Jean-Pierre Gutton, L'adoption, Paris, Publisud, 1993

${ }^{8}$ Agnès Fine, «Le don d'enfants dans l'ancienne France », in A.Fine (dir),1998, Adoptions, .op.cit, p 61-95.
} 
-...à celle d'un fils ou d'une fille à chérir

Emerge progressivement une autre finalité de l'adoption : il n'est plus seulement question pour les adoptants de rechercher un successeur légitime mais aussi un enfant à chérir. Tout au long du XIX ${ }^{\mathrm{e}}$ siècle, s'affirme l'expression d'un " désir d'enfant " et pas seulement d'un désir de descendance, tandis que la défiance à l'égard de l'intégration d'un sang étranger au sein de la famille tend à s'atténuer. La loi de 1923, votée après la Grande Guerre qui avait multiplié le nombre des orphelins, permet de combler ce désir en autorisant un couple, désormais plus jeune, - l'âge légal des adoptants ayant été abaissé de 50 à 40 ans-, à élever et adopter un enfant (et non plus un adulte). Le nombre des adoptions augmente de manière importante, et la part relative des couples parmi les adoptants augmente elle aussi régulièrement. Le contenu nouveau qu'exprime l'adoption des mineurs ne s'oppose pas à la fonction successorale de l'adoption. Ces deux finalités se recouvrent et sont difficilement dissociables.

\section{-Un enfant dont on connaît les parents}

Selon un sondage dans les archives des adoptions pratiquées dans deux départements méridionaux entre 1923 et $1960^{9}$, les adoptants étaient plutôt des personnes assez âgées, entre 40 et 70 ans, qui adoptaient des adultes qu'elles avaient généralement élevés depuis leur enfance : le plus souvent des enfants de conjoints (plus de 60\% des adoptions simples en région parisienne au début des années 60), des neveux ou des nièces (plus de $20 \%$ des cas), parfois des enfants de voisins ou d'amis, issus d'une famille nombreuse. L'adoption était « inspirée par un esprit testamentaire » dans le sens où l'adoptant, sans descendant, refusant la dispersion de ses biens, préférait les transmettre à un seul enfant, comme c'est d'ailleurs la coutume dominante dans nombre de régions françaises, en particulier dans le Midi. Si la loi prévoyait que les enfants adoptés pouvaient hériter aussi de leurs propres parents, la coutume s'y opposait. Les bénéfices patrimoniaux de ce don d'enfant devaient profiter à tous les enfants, en particulier à ceux qui restaient avec leurs parents de sang. Les futurs adoptés arrivaient chez leurs futurs parents adoptifs entre 2 et 12 ans, l'âge moyen se situant autour de 6 ou 7 ans. Ils connaissaient donc parfaitement leurs parents et, lorsqu'ils étaient les neveux ou nièces de leurs parents adoptifs, ils s'adressaient à eux avec les termes de parenté appropriés (oncle ou tante) tandis qu'ils continuaient à appeler leurs propres parents papa, maman. Dans l'adoption du neveu ou de la nièce, des relations affectives préexistaient à l'établissement de la vocation successorale de l'adopté. C'est vrai aussi des enfants de conjoints adoptés : $2 / 3$ avaient perdu l'un de leurs parents au moment de l'adoption et avaient été probablement élevés une partie de leur enfance par leur mère avec son nouveau conjoint, $1 / 3$ étaient des enfants de conjoints divorcés, et dans ce cas, leur parent avait dû consentir à leur adoption ${ }^{10}$. Souvent le parent adoptif était une femme qui, en élevant un enfant mineur, était poussée par le désir de nouer avec lui des relations affectives privilégiées. Les femmes étaient en effet nombreuses à élever seules et à adopter des enfants. Entre 1923 et 1960, sur 255 adoptions qui ont eu lieu dans le département du Tarn, 58\% ont été réalisées par des personnes seules dont les deux tiers sont des femmes, surtout des veuves, et en nombre moins important des célibataires. Les adoptés conservaient leur filiation d'origine, ils continuaient à porter le nom de leur père de naissance auquel ils ajoutaient celui de leur père adoptif. Notons que l'adoption additive, inclusive, était une forme particulièrement adaptée à l'adoption tardive, ainsi qu'à l'adoption par des personnes seules.

\section{-Un enfant sans parents proposé par l'Etat : le modèle de substitution}

\footnotetext{
${ }^{9}$ Agnès Galibert, Formes et enjeux de l'adoption ancienne, à partir d'exemples tarnais (1923-fin des années 1960), Maîtrise d'histoire sous la direction d' A.Fine, Université de Toulouse-Le Mirail , 1999

${ }^{10}$ Enquêtes menées dans la région parisienne au début des années 60 par Marie-Pierre Marmier, Sociologie de l'adoption. Etude de sociologie juridique. Thèse pour le doctorat en droit, Paris, 1969. Voir p 40 et ss
} 
Pendant la même période, apparait une nouvelle forme d'adoption, celle d'enfants petits, orphelins ou abandonnés, majoritairement adoptés par des couples stériles. Elle fonde sur le plan juridique une nouvelle finalité de l'adoption, donner une famille à un enfant qui en est dépourvu. Ces enfants sans parents font l'objet d'une législation spécifique, la légitimation adoptive, interdite aux personnes seules. En effet, en 1939, le code de la famille prévoit que seuls les couples mariés depuis plus de 10 ans sans enfants et dont l'un des deux a au moins 35 ans peuvent adopter des enfants de moins de cinq ans, abandonnés ou dont les parents sont inconnus ou décédés, en en faisant leurs enfants légitimes. La mention de la légitimation adoptive est faite en marge de l'acte de naissance de l'enfant qui a les mêmes droits et les mêmes obligations que s'il était né du mariage. L'adopté ne porte que le nom de son père adoptif $^{11}$. Une coupure définitive avec la famille naturelle est instaurée, qui sera consacrée par une nouvelle forme d'adoption, l'adoption plénière en 1966.

Cette nouvelle forme d'adoption voit le jour grâce à l'intervention d'un nouvel acteur, l'État, représenté par les services administratifs de la protection de l'enfance. Notons en effet que c'est seulement à cette époque, l'entre-deux-guerres, que l'histoire de l'abandon et celle de l'adoption, longtemps parallèles, se sont croisées de manière durable. Auparavant en effet, non seulement les enfants adoptés n'étaient pas des enfants abandonnés, mais ces derniers n'étaient pas adoptés. De fait l'adoption n'était pas pensée comme une solution à la question de l'enfance assistée.

A partir de 1923, l'État prend l'initiative de placer en adoption les enfants orphelins et abandonnés. L'échange direct entre protagonistes étant exclu, pour être adopté, un enfant doit donc d'abord être abandonné, de sorte que si l'on admet la succession de plusieurs parents dans l'histoire d'un enfant, on refuse l'idée d'une possible coexistence, non seulement en droit, mais aussi dans la réalité de la vie de l'enfant. C'est ainsi que «l'effacement de la famille d'origine " a été recherché explicitement par le législateur dès 1923 et qu'il a été érigé en principe législatif en 1939 en France avec la légitimation adoptive, puis définitivement consacré en 1966 avec la loi sur l'adoption plénière. Ces textes qui consacrent l'usage de l'adoption comme forme substitutive de procréation pour les couples sans enfant, ainsi que la conjoncture nataliste de l'époque, expliquent la multiplication du nombre des adoptions à partir de 1943, ce type d'adoption ayant rapidement pris le pas sur l'adoption classique en ce qui concerne les petits enfants. Les légitimations adoptives représentent $35 \%$ de l'ensemble des adoptions en 1952, 43\% en 1966. Les enfants adoptés sont essentiellement des pupilles de l'Etat, orphelins ou abandonnés, généralement très petits, tandis que les adoptants sont des couples stériles, heureux de satisfaire leur besoin de paternité et surtout de maternité dans les conditions les plus proches de la procréation naturelle, c'est à dire, sans la présence ou la concurrence de parents de sang. En instituant l'adoption plénière, bien distinguée dans ses effets de l'ancienne adoption qui devient désormais l'adoption simple, la loi de 1966 consacre ce nouveau modèle. Votée pour mettre un terme définitif à la possible concurrence entre parents adoptifs et parents de sang qu'avait posée de manière cruciale et très médiatisée l'affaire Novack $^{12}$, l'adoption plénière est exclusive en ce sens que tout lien légal entre l'adopté et sa famille d'origine est coupé. Les parents adoptifs deviennent les seuls et uniques parents de l'enfant adopté qui porte désormais uniquement leur nom, et dans son acte de naissance ses parents adoptifs sont désignés comme s'ils étaient ses parents de sang.

\footnotetext{
${ }^{11}$ Pour la première fois, la loi de 1949 donne aux parents adoptifs la possibilité de changer les prénoms des adoptés.

${ }^{12}$ L'affaire Novack est un conflit autour d'un enfant que ses parents naturels veulent reprendre à ses parents adoptifs.
} 
L'adoption simple des enfants passe au second plan, elle concerne essentiellement l'adoption de l'enfant de conjoint ${ }^{13}$. En quelques années, l'adoption plénière devient la forme dominante d'adoption. Aujourd'hui plus de $90 \%$ des couples qui adoptent des enfants petits, orphelins ou abandonnés français et étrangers ${ }^{14}$ usent de cette forme légale. L'adoption plénière concerne aussi les adoptants vivant seuls qui peuvent désormais être les seuls parents légaux d'un enfant, ce qui n'était pas le cas auparavant. Le parent seul ne se vit pas seulement comme un deuxième parent, partageant sa paternité ou sa maternité avec d'autres, mais comme le seul parent. Cette adoption n'a manifestement plus le même contenu que celle que nous avons décrite précédemment.

L'usage de l'institution du point de vue des parents, se combine à celui qu'en font les services de protection de l'enfance pour lui donner un contenu nouveau. F.R.Ouellette observant en anthropologue les usages de l'adoption au Québec, -mais son analyse vaut pour l'ensemble des sociétés occidentales-, a montré comment les logiques de fonctionnement des services de protection de l'enfance contribuent à forger une nouvelle définition de l'enfant et des parents ${ }^{15}$. Pour mieux préserver l'intérêt de l'enfant dont la sécurité et le développement sont compromis parce qu'il a été abandonné ou parce qu'il ne reçoit pas de ses parents les soins adéquats, les services sociaux cherchent à lui procurer, grâce à l'adoption, une famille stable avec des parents évalués pour leur capacité parentale. L'enfant est donc défini avant tout comme une personne mineure à protéger et l'adoption comme un transfert des droits et responsabilités parentales à l'égard de l'enfant. La rupture de la filiation d'origine et son impact identitaire restent dans l'ombre ; elle apparaît comme un élément secondaire dans une optique de protection centrée sur les besoins immédiats de l'enfant. Comme le souligne F.R.Ouellette, la position généalogique de l'enfant n'est pas appelée à le définir, même si l'adoption consiste bel et bien à le déloger de cette position pour l'intégrer dans un autre réseau de parenté, ce qui lui confère une nouvelle identité. Dans cette optique, l'adoption peut être valorisée même pour des enfants d'un certain âge, y compris avec filiation établie, et même pour des enfants dont les parents refusent l'adoption. Cette logique institutionnelle se heurte aujourd'hui à de nombreuses contradictions.

\section{Les transformations de l'adoption}

Le modèle de substitution propre aux pays occidentaux est ébranlé aujourd'hui par trois facteurs : l'origine étrangère de la majorité des adoptés qui rend l'adoption visible, la pénurie relative des enfants adoptables qui rend plus fréquente l'adoption d'enfants un peu plus âgés, le développement d'une nouvelle forme d'adoption, l'adoption «ouverte », dans laquelle parents de sang et parents adoptifs ont des communications les uns avec les autres avant et/ou après l'adoption ${ }^{16}$. L'adoption « ouverte» est pratiquée depuis deux décennies aux EtatsUnis, depuis quelques années au Canada et dans d'autres pays européens et tend à remplacer

\footnotetext{
${ }^{13}$ Avec l'augmentation des recompositions familiales après divorce, le nombre des adoptions simples des enfants de conjoints) a beaucoup augmenté passant d'environ 3000 par an dans les années 1985 à plus de 6000 aujourd'hui.

14 En 1992, près de 93\% des adoptions plénières concernant des enfants étrangers ou pupilles de l'Etat étaient réalisées par des couples. cf Infostat Justice, septembre 1996, n46

${ }^{15}$ Françoise-Romaine Ouellette, «L'adoption face aux redéfinitions de la famille et de l'institution généalogique », A.Fine et C.Neirinck (dir), Parents de sang, parents adoptifs, op.cit, p 325-341; .voir aussi du même auteur 1996, L'adoption. Les acteurs et les enjeux autour de l'enfant. Sainte Foy, Les Presses de l'université de Laval. ; avec L.R.Frigault, 1996, Les adoptions internationales au Québec, Montréal, INRS, Culture et Société ; avec Catherine Méthot, L'adoption tardive internationale. L'intégration familiale de l'enfant du point de vue des parents et des grands-parents, Montréal INRS-Culture et Société.

${ }^{16}$ Sur les aspects historiques, juridiques et anthropologiques de l'adoption ouverte et fermée, voir A.Fine et C.Neirinck, 2000, Parents de sang, parents adoptifs. Approches juridiques et anthropologiques de l'adoption. France, Europe, USA, Canada, Paris, LGDJ.
} 
l'adoption «fermée », fondée sur l'anonymat des parties impliquées et le secret absolu des dossiers d'adoption comme des actes d'état civil originels. Elle s'est imposée peu à peu sous la pression de groupes d'adoptés luttant pour leurs droits à connaître leur histoire, les circonstances de leur naissance et de leur abandon, éventuellement l'identité de leurs parents de sang. En France ces mouvements ont obtenu la création en 2002 du $\mathrm{CNAOP}^{17}$, organisme centralisé, censé aider les adoptés dans leurs recherches personnelles. L'adoption ouverte concerne essentiellement l'adoption « interne » et assez peu l'adoption internationale.

Cette dernière, qui concernerait aujourd'hui dans le monde entre 15000 et 20000 enfants, fait l'objet d'une régulation de plus en plus importante, pour éviter les dérives que ne manque pas de provoquer le déséquilibre croissant entre le nombre des parents souhaitant adopter et le nombre des enfants adoptables. La Convention internationale de la Haye, votée en 1993 et ratifiée en 2006 par une quarantaine de pays, a défini un certain nombre de principes à respecter pour permettre l'adoption internationale d'un enfant. L'un d'eux relatif au consentement «éclairé » des parents à l'abandon de leurs enfants concerne directement les anthropologues qui contribuent à mieux l'évaluer. Ainsi à propos de la situation brésilienne, Claudia Fonseca ${ }^{18}$ a mis en évidence la manière dont la circulation informelle des enfants dans les familles pauvres se confronte aux nouvelles lois et aux pratiques étatiques conformes aux normes internationales. Des enfants placés en orphelinat en temps de crise, sont considérés comme délaissés et donnés en adoption internationale, sans l'accord réel de leur mère, pour laquelle ce placement est provisoire. Par ailleurs, de nombreux pays ne connaissent que l'adoption simple qui, n'entraînant pas la rupture totale des liens de filiation entre parents et enfants, ajoute la filiation adoptive à la filiation d'origine. Or dans les pays qui ne connaissent qu'une seule forme d'adoption, comme le Canada ou les USA, ces adoptions simples sont transformées d'autorité en adoptions plénières dans le pays d'accueil. De même peut-on parler de consentement éclairé des parents d'origine lorsque l'adoption n'était pas souhaitée a priori par les personnes concernées ? Chantal Collard note a propos des adoptions d'enfants haïtiens au Québec, que la circulation informelle des enfants entre familles apparentées est amenée à se couler dans un moule légal lorsque les familles sont séparées par des barrières nationales. Elle est alors soumise au contrôle de l'immigration et beaucoup des résidents étrangers sont obligés d'user de l'adoption pour accueillir un enfant de leur parenté chez eux. Ainsi jouent les décalages culturels qui, combinés aux rapports de domination économique entre sociétés, interdisent les solutions traditionnelles qui permettaient lors d'une crise familiale (décès, maladie ou autres revers) que des enfants soient élevés par des membres de leur parenté. L'anthropologue met en évidence les effets juridiques de la rupture des liens d'origine et de la nouvelle filiation : des tantes, des cousines, des demisœurs deviennent des mères adoptives, perturbant de manière extraordinaire la généalogie des adoptés ${ }^{19}$.

Aujourd'hui sous l'effet de la mondialisation des échanges et de l'impact des organisations internationales dans les pays donneurs, on assiste aussi à des mutations importantes des formes traditionnelles d'adoptions. Alors qu'en Polynésie française, par exemple, les parents qui n'avaient pas les moyens d'élever leur enfant le donnaient à un couple proche, depuis une vingtaine d'années, ils le donnent à des couples métropolitains, qui

\footnotetext{
${ }^{17}$ Conseil National pour l'accès aux origines personnelles. La loi du 22 janvier 2002 n'a pas pour autant levé le secret sur la naissance dans l'intérêt de la mère.

${ }^{18}$ Claudia Fonseca, 1999, «Circulation d'enfants ou adoption : les enjeux internationaux de la filiation adoptive », Droit et Cultures, vol 38, 2, p 136-167 et « La circulation des enfants pauvres au Brésil. Une pratique locale dans un monde globalisé », Anthropologie et Sociétés, vol 24, 3, p 53-73;

${ }^{19}$ Chantal Collard, 2004, « La politique du fosterage et l'adoption internationale en Haïti », in I.Leblic (éd), De l'adoption, op.cit, p 239-267 ; voir aussi «Les adoptions internationales d'un enfant apparenté au Québec », in Françoise-Romaine Ouellette, Renée Joyal et Roch Hurtebise, 2005, Familles en mouvance : quels enjeux éthiques? Presses de l'Université Laval, Coll.Culture et Société, p 121-140
} 
souvent n'ont qu'une compréhension partielle des coutumes locales en la matière. Des malentendus apparaissent mais aussi des transformations dans les pratiques ici et là-bas. Le choix de donner un enfant à des parents d'origine occidentale répond à l'espoir de ses parents de lui donner un statut social privilégié ainsi qu'à celui de recevoir éventuellement des retombées économiques en échange. Du côté des parents adoptifs métropolitains, une attention plus grande est portée désormais au maintien des liens avec les parents de naissance, ce qui n'est pas sans effet sur les pratiques françaises d'adoption, encore largement attachées au secret sur les filiations de naissance des enfants adoptés. 